\title{
Kidney Re-Transplantation During Childhood Feasibility and Outcomes
}

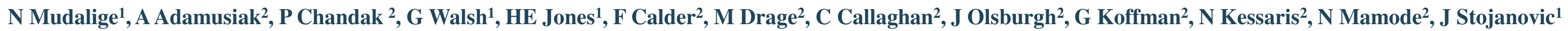
Evelina London Children's Hospital'1, Guy's Hospital, London²

\section{INTRODUCTION}

End stage renal disease occurs at an incidence of 5.5 children per million age matched children within Europe[1]. Transplantation remains the gold standard therapy with a vast increase in quality of life and this is taking place in ever younger children [2].

Large studies suggest that $21.8 \%$ of primary paediatric transplants fail in childhood $[3,4]$. Others report that, in children with $\geq 2$ transplants, the second graft survival to be longer than the first (8.5 vs 4.5 years)[5]. However, characteristics of children whose transplant fails in childhood are poorly reported. This study looked into these characteristics.

\section{METHODS}

All children <18 years of age and transplanted at Evelina London between 01/2003 - 09/2015 were included. A total of 171 renal transplants were performed; 9 of which were re-transplants. We performed a retrospective analysis of these 9 patients. Mean follow up period was 42.8 months (range 18-65).

\section{RESULTS}

$\underline{\text { Patient characteristics at the time of first transplant (Table 1) }}$

\section{- $\quad 45 \%$ were pre emptive transplants.}

- $66 \%$ were small recipients $(<20 \mathrm{~kg})$.

- $\quad 88 \%$ received organ from a deceased donor.

- $\quad$ Two grafts were lost due to vascular complications $(<20 \mathrm{~kg})$.

- $\quad$ Average graft survival 8.9years (range $2 \mathrm{~m}-16 \mathrm{yrs}$ ).

- $\quad$ All patients were CMV and EBV naïve.

- 1 child developed PTLD - 100\% patient/graft survival.

- $\quad 89 \%$ are males.

\begin{tabular}{|c|c|c|c|c|c|c|c|c|}
\hline & $\begin{array}{c}\text { Etiology of } \\
\text { ESRD }\end{array}$ & $\begin{array}{l}\text { Age } \\
\text { (yrs) }\end{array}$ & Donor & $\begin{array}{l}\text { Weight } \\
\text { (kg) }\end{array}$ & $\begin{array}{l}\text { Arterial } \\
\text { Anast- } \\
\text { omosis }\end{array}$ & $\begin{array}{c}\text { Venous } \\
\text { Anasto- } \\
\text { mosis }\end{array}$ & $\begin{array}{l}\text { Cause for graft } \\
\text { failure }\end{array}$ & $\begin{array}{l}\text { Graft survival } \\
\text { (days) }\end{array}$ \\
\hline 1 & CNS & 2 & LRD & $<20$ & $A A$ & IVC & $\begin{array}{l}\text { Recurrent } \\
\text { rejections }\end{array}$ & 126 \\
\hline 2 & $\begin{array}{l}\text { Cortical } \\
\text { necrosis }\end{array}$ & 1.7 & DD & $<20$ & AA & IVC & $\begin{array}{l}\text { Recurrent } \\
\text { rejections }\end{array}$ & 3608 \\
\hline 3 & VUR & 2.7 & DD & $<20$ & AA & IVC & $\begin{array}{l}\text { Recurrent } \\
\text { rejections }\end{array}$ & 4325 \\
\hline 4 & VUR & 4.3 & DD & $>20$ & $\mathrm{ClA}$ & CIV & $\begin{array}{c}\text { Chronic } \\
\text { active AMR }\end{array}$ & 2510 \\
\hline 5 & VUR & 3.8 & DD & $>20$ & $\mathrm{ClA}$ & IVC & $\begin{array}{l}\text { Renal arterial } \\
\text { thrombosis }\end{array}$ & 12 \\
\hline 6 & CNS & 2 & DD & $<20$ & AA & IVC & $\begin{array}{l}\text { Renal venous } \\
\text { thrombosis }\end{array}$ & 2 \\
\hline 7 & $\begin{array}{l}\text { Dysplasia } \\
\& \text { VUR }\end{array}$ & 7.5 & DD & $<20$ & AA & IVC & $\begin{array}{c}\text { Chronic } \\
\text { active AMR }\end{array}$ & 3258 \\
\hline 8 & $\begin{array}{l}\text { Dysplasia } \\
\text { \& VUR }\end{array}$ & 1.7 & DD & $<20$ & $A A$ & IVC & $\begin{array}{c}\text { Chronic } \\
\text { active AMR }\end{array}$ & 5852 \\
\hline 9 & FSGS & 9 & DD & $>20$ & $\mathrm{CIA}$ & CIV & $\begin{array}{c}\text { FSGS } \\
\text { recurrence }\end{array}$ & 113 \\
\hline
\end{tabular}

Table 1. Characteristics of 1 st transplant. CNS-congenital nephrotic syndrome; VUR-vesico-ureteric reflux; LRD-living related donor; DD-deceased donor; AA-abdominal aorta; CIA-common iliac artery; IVC-inferior vena cava; CIVcommon iliac vein; ESRD-End stage renal disease;AMR - antibody mediated rejection;FSGS-focal segmental glomerular sclerosis.

\section{RESULTS}

\section{$\underline{\text { Patient characteristics at the time of } 2^{\text {nd }} \text { transplant (Table 2) }}$}

- $55 \%$ were pre-emptive re-transplants.

- $50 \%$ HLA sensitised (1 with cRF>85\%).

- $22 \%$ were small recipients $(<20 \mathrm{~kg})$.

- 2 grafts were re-anastomosed to the aorta \& IVC.

- $55 \%$ received organ from a deceased donor.

- All received MMF, tacrolimus and corticosteroids.

- $\quad 62 \% \mathrm{CMV}$ and $50 \% \mathrm{EBV}$ were seropositive.

- $\quad 3 / 9$ experienced $2^{\text {nd }}$ graft failure (one received a $3^{\text {rd }}$ transplant, one is currently on dialysis and one on the waiting list).

Graft survival for children with a single transplant compared with those re-transplanted at 3 year follow up $96 \%$ vs $89 \%(p=0.21)$

\begin{tabular}{cccccc}
\hline & $\begin{array}{c}\text { Weight } \\
\mathbf{( k g )}\end{array}$ & $\begin{array}{c}\text { Arterial } \\
\text { Anasto- } \\
\text { mosis }\end{array}$ & $\begin{array}{c}\text { Venous } \\
\text { Anasto- } \\
\text { mosis }\end{array}$ & $\begin{array}{c}\text { Aetiology of graft Graft survival } \\
\text { failure }\end{array}$ & (days) \\
\hline 1 & 17.3 & AA & IVC & $\begin{array}{c}\text { Chronic active } \\
\text { AMR }\end{array}$ & 3968 \\
\hline 2 & 25 & CIA & EIV & $\begin{array}{c}\text { BK nephropathy } \\
\text { and rejections }\end{array}$ & 962 \\
\hline 3 & 54.5 & CIA & EIV & N/A & Functioning \\
\hline 4 & 65.2 & CIA & CIV & N/A & Functioning \\
\hline 5 & 21.9 & AA & IVC & N/A & Functioning \\
\hline 6 & 18 & AA & IVC & $\begin{array}{c}\text { TMA/Chronic } \\
\text { active AMR }\end{array}$ & 2114 \\
\hline 7 & 50 & CIA & CIV & N/A & Functioning \\
\hline 8 & 55.9 & CIA & CIV & N/A & Functioning \\
\hline 9 & 53.5 & CIA & CIV & N/A & Functioning \\
\hline
\end{tabular}

Table 2. Characteristics of 2 nd transplant. TMA-thrombotic microangiopathy.

\section{CONCLUSIONS}

-We report a low rate at $5.2 \%$ of kidney re-transplantation in childhood in a large paediatric cohort.

-The majority of recipients were small at the time of first transplant and were CMV/EBV naïve. A large number of first grafts in this cohort were lost due to chronic active antibody mediated rejection.

-The second graft anastomosis to the aorta and IVC is feasible.

-There was no difference in graft survival at three years between first transplant and those who were re-transplanted. Importantly one patient received 3 kidney transplants by the age of eight.

-Re-transplants are achievable but associated with surgical, immunological and viral challenges.

- Limitations of this study include that it is a retrospective single centre analysis with a relatively small sample size. Larger studies are needed to further define this unique population.

11] Chesnaye, N. et al. Demographics of padiatric renal replacement therapy in Europe: a report of the ESPN/ERA-EDTA registry. Pediatr. Nephrol. 29, 2403-2410 (2014)

[2] Gondos, A., Döhler, B., Brenner, H. \& Opelz, G. Kidney graft survival in Europe and the United States: strikingly different long-term outcomes. Transplantation 95, 267-274 (2013).

[3] FALGER J, te al Outcome after renal transplantation. Part I: intellectual and motor performance Pediatr Nephrol 2008 Aug:23(8):1339-1345

[4] Foster, B. J. Heightened graft failure risk during emerging adulthood and transition to adult care. Pediatr. Nephrol. 30, 567-576 (2015).

[5] Van Arendonk KJ, James NT, et al. Age at Graft Loss after Pediatric Kidney Transplantation: Exploring the High-Risk Age Window. Clin J Am SocNephrol. 2013 Jun;8(6):1019-1026. 